\title{
Estilos de aprendizaje de estudiantes wayuu en universidades públicas del departamento de La Guajira, Colombia
}

\author{
Learning styles of wayuu students in public universities \\ of the department of La Guajira, Colombia
}

\section{Remedios Pitre-Redondo' \\ Norma Margarita Sánchez-Martínez ${ }^{2}$ Hugo Gaspar Hernández-Palma ${ }^{3}$}

Recibido: mayo 15 de 2020

Aceptado: septiembre 07 de 2020

\section{Resumen}

El presente artículo busca identificar los estilos de aprendizaje que se observan en los estudiantes de la etnia wayuu, de las universidades públicas del departamento de la Guajira, Colombia. En el contexto metodológico, el estudio se caracteriza como descriptivo con enfoque cuantitativo. La muestra estuvo constituida por 210 estudiantes seleccionados mediante un muestreo intencional, de las instituciones: Universidad de la Guajira (Uniguajira) y Universidad Nacional Abierta y a Distancia (UNAD). Los resultados demuestran que los estilos de aprendizaje predominantes, son: activo, reflexivo y pragmático; y en menor proporción, se hace presente el estilo teórico. Se concluye que los estilos de aprendizaje no resultan excluyentes entre sí, ya que puede producirse una combinación o alternancia de estos, con el fin de lograr el aprendizaje de alto valor en diferentes momentos, contextos o circunstancias.

Palabras clave: estilos de aprendizaje, universidades públicas, educación superior, etnia Wayuu, pueblos indígenas.

\begin{abstract}
This article is addressed to identify the learning styles observed in students of the Wayuu ethnic group, from public universities in the department of La Guajira, Colombia. From the methodological context, the study is characterized as descriptive with a quantitative approach. The sample consisted of 210 students selected through an intentional sampling of the institutions: Universidad de la Guajira (Uniguajira), and Universidad Nacional Abierta y a Distancia (UNAD). The results show that: active, reflective and pragmatic learning styles, predominate in the students under study; and to a lesser extent, the theoretical style is present. It is concluded that learning styles are not mutually exclusive, since a combination or alternation of these can occur in order to achieve high value learning, at different times, contexts or circumstances.
\end{abstract}

Keywords: learning styles, public universities, higher education, Wayuu ethnic group, indigenous people.

1 Economista, Doctora en Ciencias Gerenciales, Universidad de La Guajira, Riohacha, Colombia. E-mail: rpitre@uniguajira.edu.co ORCID: https://orcid.org/0000-0001-7373-1101

2 Trabajadora Social, Magíster en Gerencia de Proyectos de Investigación y Desarrollo, Universidad de La Guajira, Riohacha, Colombia. E-mail: Nmsanchez@uniguajira.edu.co

ORCID: https://orcid.org/0000-0001-8216-0195

3 Ingeniero Industrial, Magíster en Sistemas de Gestión, Universidad del Atlántico, Barranquilla, Colombia. E-mail: hugoghernandezpalma@ gmail.com

ORCID: https://orcid.org/0000-0002-3873-0530 


\section{Introducción}

El individuo, por naturaleza, es un ser racional que se encuentra en evolución permanente y parte sustancial de su desarrollo radica en su capacidad cognitiva de aprender. De acuerdo con diversos autores e investigadores, a lo largo del tiempo se han profundizado interrogantes relativas a cómo, qué, cuándo se aprende, dando lugar al surgimiento de diversas teorías contemporáneas ampliamente conocidas en el campo educativo, a saber: el constructivismo (Montealegre, 2016), el aprendizaje significativo (DeBoer, 2019), aprendizaje por descubrimiento (Pingo, Muñoz, Reyes \& Polo, 2020), el cognitivismo (Bertrán, 2018) hasta su aplicación enfoques educativos modernos como la formación por competencias (Niebles, Martínez-Bustos, \& Niebles-Núñez, 2020).

En este sentido, también es menester preguntarse: ¿todos los individuos aprenden de igual manera?. Sintetizando lo expresado por los teóricos previamente mencionados, al intentar definir en qué consiste el aprendizaje, se señala que es un proceso cognitivo, dinámico y continuo, que se puede llevar a cabo al memorizar, al incrementar el conocimiento, igualmente cuando el mismo es aplicado, producto de la experiencia, o bien como una causa del cambio de actitud lo cual conlleva al desarrollo personal.

Asimismo, el proceso de aprendizaje puede generarse de manera formal o informal, dentro o fuera del ambiente educativo; se plantea, por lo tanto, el logro de habilidades de pensamiento y abstracción. En tal sentido, Contreras et al. (2017), se refieren al aprendizaje como a una práctica, que promueve: la ejercitación de destrezas básicas, la posterior internalización de estas y la comprensión de conceptos.

Ahora bien, este trabajo tiene por objetivo identificar los estilos de aprendizaje que se observan en los estudiantes de la etnia Wayuu, de las universidades públicas del departamento de La Guajira, Colombia, específicamente de la Universidad de La Guajira (Uniguajira), y la Universidad Nacional Abierta y a Distancia (UNAD). El interés en la etnia Wayuu, radica en que este segmento de la población indígena de Colombia, tiene rasgos: cognitivos, fisiológicos, hereditarios, culturales y familiares, que los hacen particulares y que indudablemente inciden en el proceso de aprendizaje que los mismos desarrollan.

En cuanto a la población objeto de estudio, se destaca que para la Organización Nacional Indígena de Colombia (ONIC, 2019), y para el Ministerio de Cultura de Colombia, con basamento en el censo DANE 2005 (Mincultura, 2019), se reconoce a la etnia Wayuu como el pueblo indígena de mayor cantidad de población del país, concentrado en el departamento de La Guajira, en el cual habita el $98,03 \%$ del total. Es una comunidad considerada cultural y socialmente fuerte, que ha logrado articularse de manera exitosa a procesos regionales y nacionales, en los ámbitos: económico, cultural, social y político, sin perder su quehacer tradicional ni evidenciar debilitamiento de su cultura (Mincultura, 2019).

Lo anterior tiene impacto en las universidades objeto de esta investigación, ya que la misión de Uniguajira (2019), establece:

(...) Formar profesionales íntegros que perciban, aprehendan, generen, apliquen y transformen saberes, conocimientos en un marco de diversidad cultural a través de las actividades que le son propias (docencia, investigación, proyección social y extensión) para satisfacer las necesidades de desarrollo sostenible y sustentable de la sociedad guajira y de su entorno, con especial énfasis en el autorreconocimiento de las condiciones de multiculturalidad, con miras al desarrollo de la personalidad integral de todos sus actores institucionales y de la comunidad en general, para alcanzar condiciones de interculturalidad.

Así mismo, la UNAD (2019), describe su misión, como:

(...) Contribuir a la educación para todos a través de la modalidad abierta, a distancia y en ambientes virtuales de aprendizaje, 
mediante la acción pedagógica, la proyección social, el desarrollo regional y la proyección comunitaria, la inclusión, la investigación, la internacionalización y las innovaciones metodológicas y didácticas, con la utilización de las tecnologías de la información y las comunicaciones para fomentar y acompañar el aprendizaje autónomo, generador de cultura y espíritu emprendedor que, en el marco de la sociedad global y del conocimiento, propicie el desarrollo económico, social y humano sostenible de las comunidades locales, regionales y globales, con calidad, eficiencia y equidad social.

\section{Marco teórico y metodología}

\subsection{Estilos de aprendizaje}

En el proceso de aprendizaje, es pertinente destacar la interacción constante a la que está sometido el ser humano en relación con su medio ambiente o contexto, del cual recibe información, la convierte en conocimiento por medio de la codificación, el almacenamiento y la recuperación de esta data, la cual luego utiliza y transforma (Bertrán, 2018). Esta experiencia se puede desarrollar como producto de la observación, repetición, descubrimiento o recepción.

En este artículo, se realiza una aproximación para identificar los estilos de aprendizaje que se demuestran en los estudiantes universitarios de la etnia wayuu. Los estilos de aprendizaje, según Cortés (2017), se definen como los rasgos cognitivos, afectivos y fisiológicos que sirven como indicadores relativamente estables, de cómo los discentes perciben, interaccionan y responden a sus ambientes de aprendizaje. Para alcanzar el propósito de este artículo, se desarrolló una revisión teórica de fuentes primarias y secundarias, que incluyen investigaciones relacionadas con el tema de los estilos de aprendizaje, tales como las de Gutiérrez, García y Melaré (2012), y Malacapay (2019).
En cuanto al desarrollo de instrumentos de diagnóstico para perfilar los estilos de aprendizaje, se dice que los mismos se iniciaron desde la década de los años 60 del siglo pasado. No obstante, para los fines de esta investigación, se asumen como principales referentes a Honey y Mumford (2000), quienes formularon sus aportes a partir de la propuesta de Kolb (Martin, Sperling \& Newton, 2020). La evaluación de los estilos se erige sobre la influencia del contexto para aprender, considerando que este proceso se desarrolla de manera cíclica en fases. Posteriormente, sobre la base de los planteamientos de los precitados autores, Honey y Mumford realizaron una adaptación de los estilos, y desarrollaron un instrumento de diagnóstico para perfilar los mismos (Cortés, 2017).

Por otro lado, García, Santizo y Alonso (2009), realizaron una investigación donde compilan las posturas de diferentes autores, encontrando veintiún (21) variables que influyen en la manera de aprender. Con base en las teorías de aprendizaje de tipo cognitivo, Honey y Mumford (2000), expresan que los estilos de aprendizaje son algo así como la interiorización por parte de cada sujeto de una etapa determinada del ciclo. Los estilos, para estos autores, son cuatro: activo, reflexivo, teórico y pragmático, que se pueden describir de la siguiente manera:

i) Los activos se implican plenamente y sin prejuicios en nuevas experiencias. Son de mente abierta, nada escépticos y acometen con entusiasmo las tareas nuevas. Son gentes del aquí y ahora y les agrada vivir nuevas experiencias. Sus días están llenos de actividad.

ii) Los reflexivos consideran las experiencias y las observan desde diferentes perspectivas.

iii) Los teóricos adaptan e integran las observaciones dentro de teorías lógicas y complejas. Enfocan los problemas de forma vertical escalonada, por etapas lógicas. Tienden a ser perfeccionistas. Integran los hechos en teorías coherentes. Les gusta analizar y sintetizar. 
iv) Los pragmáticos tienen su punto fuerte en la aplicación práctica de las ideas. Descubren el aspecto positivo de las nuevas ideas y aprovechan la primera oportunidad para experimentarlas. Les agrada actuar rápidamente y con seguridad con aquellas ideas y proyectos que les atraen.

El conocimiento del estilo de aprendizaje presenta preeminencia en el ámbito educativo, pues el mismo permite una mejor comprensión de las características de cada estudiante, de la manera en la cual éste aborda la realidad y cómo aprende, lo que, sin duda, redunda en el éxito del proceso de enseñanza-aprendizaje. Para el docente constituye una fortaleza el saber al respecto de estos estilos de aprendizaje, ya que con ellos puede adecuar las estrategias de enseñanza y evaluación, además de promover aquellos estilos menos desarrollados y potenciar aún más los estilos predominantes.

En este sentido, Rodríguez (2017), señala que el enfoque de Honey y Mumford, invita a la reflexión en el aula de clase, ya que tanto profesores como estudiantes deben analizar y reestructurar sus roles ante el proceso de enseñanzaaprendizaje, especialmente en áreas reconocidas por su dificultad para lograr el aprendizaje. El profesor debe planear diversas actividades que estimulen a los estudiantes a hacer el recorrido por los cuatro estilos, que aseguran un aprendizaje sin desconocer las particularidades de cada persona; y a su vez, los estudiantes deben diseñar estrategias de estudio con las que se acomoden al proceso.

\subsection{Metodología}

El estudio se realizó siguiendo un enfoque cuantitativo, de acuerdo con la naturaleza de los datos recolectados, en los que para probar hipótesis se hace uso de la medición numérica y el análisis estadístico, con el fin de establecer pautas de comportamiento y probar teorías. Hernández, Fernández y Baptista (2014), plantean que el enfoque cuantitativo busca compilar la información obtenida durante la investigación, analizarla y convertir este resultado en la interpretación de los patrones conductuales del fenómeno y establecer veracidad de postulados. El estudio tiene un alcance descriptivo, lo que conlleva a especificar propiedades y características importantes de cualquier fenómeno que se analice; describe tendencias de un grupo o población. También se definen el análisis y los procesos que se involucrarán en la investigación (Hernández et al., 2014).

En cuanto a la población y muestra, los sujetos de investigación participantes fueron los estudiantes de la etnia Wayuu, de las Universidades Públicas del Municipio de Riohacha, Distrito Especial Turístico y Cultural, Departamento de La Guajira, Colombia. Específicamente, se tomaron estudiantes de la Universidad de La Guajira (Uniguajira), y de la Universidad Nacional Abierta y a Distancia (UNAD), pertenecientes a las diferentes carreras profesionales y cursando distintos semestres académicos. La población estuvo compuesta por 1987 estudiantes de Uniguajira, y por 375 de la UNAD. La muestra se calculó haciendo uso de la ecuación 1, correspondiente a la fórmula de muestreo planteada por Hernández et al. (2014):

$$
n=\frac{Z^{2} * p * q * N}{(N-1) * E^{2}+p * q * Z^{2}}(1)
$$

Donde Z obedece al nivel de confianza (95\%), mientras que $E$ es el límite aceptable por error muestral (5\%), con la porción positiva de elementos que presentan la característica en estudio p, junto con la porción que no lo presenta q. Arrojando como resultados una muestra de 319 individuos para Uniguajira y 189 para la UNAD, respectivamente. No obstante, de las muestras obtenidas para cada universidad, se seleccionó a juicio de los investigadores una muestra intencional (Arias, 2012), de 105 estudiantes wayuu por cada universidad, para un total definitivo de 210 individuos.

La técnica que sustentó la fase de campo, y de la cual dependió la estrategia de recolección de datos en esta investigación, fue la encuesta. En este caso, se hizo uso de un cuestionario 
validado por cinco expertos del área educativa, con perfil de docente. Dicho cuestionario está conformado por un conjunto de preguntas respecto a los estilos de aprendizaje, en el cual se enunciaron afirmaciones que cuentan con un valor cuantificable, a través de una escala Likert, la cual incluye las categorías: casi nunca, nunca, a veces, casi siempre, y siempre.

El procedimiento seguido en la investigación, se soporta en la siguiente ruta: el planteamiento de la situación objeto de investigación, la revisión de las fuentes bibliográficas relativas al tema en estudio, la definición de la población y muestra, el diseño, validación y aplicación del instrumento de recolección de datos, el procesamiento y análisis de la información y la presentación de las principales conclusiones. El análisis de datos se hizo con estadística inferencial, la cual permitió describir los valores obtenidos para cada una de las interrogantes referidas a los tipos de aprendizaje, a través de la distribución de frecuencias absolutas (fa), y frecuencias relativas (\%), y medias aritméticas como medida de tendencia central. El procesamiento se realizó por medio del software SPSS versión 24.

\section{Resultados y discusión}

\subsection{Estilos de aprendizaje en la etnia Wayuu}

Según Cortés (2017), las personas con un estilo de aprendizaje activo, se caracterizan por ser individuos que: se implican plenamente en nuevas experiencias, acometen ideas nuevas con entusiasmo y se aburren de los plazos largos. Son improvisadores, descubridores, arriesgados, espontáneos, creativos y novedosos.

En relación con el estilo de aprendizaje activo, al consultar a los estudiantes de la etnia wayuu, si se encuentran en constante búsqueda de nuevas actividades en sus procesos de aprendizaje que les permitan obtener nuevos conocimientos, la valoración siempre fue la más representativa en ambas universidades: $39 \%$ en Uniguajira y $78,1 \%$ en la UNAD. La valoración casi siempre, representa el $21,9 \%$ en Uniguajira y en la UNAD $19 \%$. Sin embargo, se evidencia un nivel neutral calificado con a veces, de $38,1 \%$ en Uniguajira y $1,9 \%$ en la UNAD. Las opciones casi nunca: $1 \%$ en Uniguajira y $0 \%$ en la UNAD, y nunca: $0 \%$ en Uniguajira y $1 \%$ en la UNAD, representan valores de poca significancia (Tabla 1).

Tabla 1. Aprendizaje activo.

\begin{tabular}{|c|c|c|c|c|c|c|}
\hline Indicador & \multicolumn{4}{|c|}{ Ítem } & \multicolumn{2}{|c|}{$\begin{array}{l}\text { Media del indicador } \\
\qquad 4,3 \%\end{array}$} \\
\hline \multirow[t]{2}{*}{ Aprendizaje activo } & \multicolumn{4}{|c|}{$\begin{array}{l}\text { ¿Está usted en constante búsqueda de nuevas activi- } \\
\text { dades en sus procesos de aprendizaje que le permitan }\end{array}$} & \multirow{2}{*}{\multicolumn{2}{|c|}{ Total }} \\
\hline & \multicolumn{4}{|c|}{ obtener nuevos conocimientos? } & & \\
\hline Universidad & & Uniguajira & & UNAD & & \\
\hline Media & & $3.9 \%$ & & $4.7 \%$ & & \\
\hline \multirow{2}{*}{ Alternativas } & \multirow{2}{*}{$\mathrm{Fa}$} & $\mathrm{Fr}$ & \multirow{2}{*}{$\mathrm{Fa}$} & $\mathrm{Fr}$ & $\mathrm{Fa}$ & $\mathrm{Fr}$ \\
\hline & & $\%$ & & $\%$ & Acumulada & Acumulada\% \\
\hline Casi nunca & 1 & $1 \%$ & 0 & $0 \%$ & 1 & $0,47 \%$ \\
\hline Nunca & 0 & $0 \%$ & 1 & $1 \%$ & 1 & $0,47 \%$ \\
\hline$A$ veces & 40 & $38,1 \%$ & 2 & $1,9 \%$ & 42 & $20,0 \%$ \\
\hline Casi siempre & 23 & $21,9 \%$ & 20 & $19 \%$ & 43 & $20,47 \%$ \\
\hline Siempre & 41 & $39 \%$ & 82 & $78,1 \%$ & 123 & $58,57 \%$ \\
\hline Totales & 105 & $100,00 \%$ & 105 & $100,00 \%$ & 210 & $100,00 \%$ \\
\hline
\end{tabular}


En la tabla 1 se observa que, de los 210 estudiantes indígenas encuestados, el 58,57\% siempre está en constante búsqueda de nuevas actividades que les generen nuevos conocimientos, mientras que el $20,47 \%$ casi siempre está en búsqueda de actividades que les faciliten la obtención de conocimiento. No obstante, el $20 \%$ de los encuestados reconoce que a veces, el $0,47 \%$ nunca (UNAD), y el $0,47 \%$ casi nunca están en búsqueda de actividades novedosas para adquirir conocimiento. La sumatoria de estas tres opciones de respuesta, representa el $41,4 \%$.

Por otra parte, se determinó la media de los datos arrojados por los estudiantes que participaron en la encuesta en cada universidad: la media en Uniguajira fue de 3,9\% (porcentaje moderado), mientras que en la UNAD fue de $4,7 \%$ (porcentaje alto). El valor total de la media del indicador, fue de 4,3\%; de acuerdo con la significación de un rango del 1 al 5 , se considera un porcentaje alto, en cuanto al estilo de aprendizaje activo y en cuanto a la búsqueda de nuevas actividades, en los procesos de obtener conocimiento por parte de los estudiantes (Tabla 1).
Según Cortés (2017), a los individuos con estilo de aprendizaje reflexivo: les gusta observar las experiencias desde distintas perspectivas, recogen datos y los analizan antes de llegar a una conclusión. Son ponderados, concienzudos, repetitivos, analíticos, asimiladores y prudentes.

Respecto al estilo de aprendizaje reflexivo, se advierte en la Tabla 2 que, al preguntar sobre la obtención de conocimiento, considerando: todas las alternativas posibles, las experiencias propias y las de otros, y la observación de las diferentes perspectivas o enfoques, la valoración más representativa, fue siempre con $70,5 \%$ en la UNAD, y casi siempre con $51,4 \%$ en Uniguajira. La siguiente valoración fue siempre con el $22,8 \%$ en Uniguajira, y casi siempre con el $25,7 \%$ en la UNAD. Entre los valores menos representativos, se encuentran las opciones a veces, con $23,9 \%$ en Uniguajira, y un $3,8 \%$ en la UNAD; y casi nunca con 1,9\% en Uniguajira, y $0 \%$ en la UNAD.

Tabla 2. Aprendizaje reflexivo.

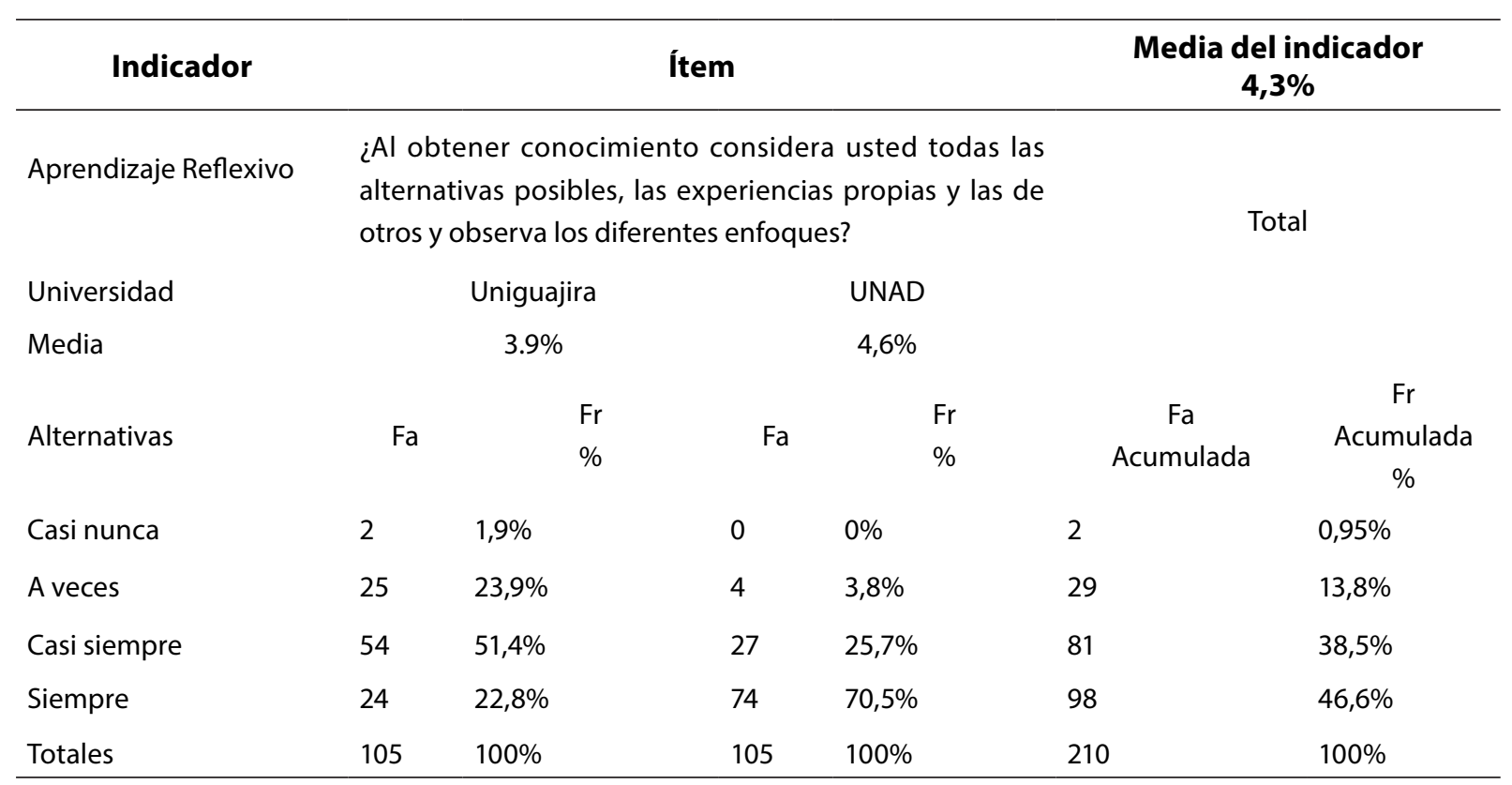


De los 210 estudiantes indígenas encuestados, el $46,6 \%$ siempre considera todas las alternativas posibles, las experiencias y los diferentes enfoques al obtener conocimiento; mientras que el $38,5 \%$ casi siempre considera los aspectos antes mencionados. En menores valores: 13,8 ) a veces, y $0,95 \%$ casi nunca (Tabla 2 ). En relación con la media de los datos arrojados para este ítem, se observa un valor de 3,9\% para Uniguajira (porcentaje moderado), mientras que para la UNAD es de 4,6\% (porcentaje alto). El valor de la media del indicador, contó con una puntuación del $4,3 \%$; de acuerdo con la significación en un rango del 1 al 5, se considera un porcentaje alto en cuanto a: valorar todas las alternativas posibles, las experiencias y los diferentes enfoques antes de realizar una actividad (Tabla 2 ).

En este punto, se puede decir que los datos revelan que los estudiantes wayuu de la UNAD, poseen el estilo de aprendizaje reflexivo en un porcentaje más alto que los de Uniguajira. Igualmente, se infiere que cierta cantidad de los estudiantes participantes en la encuesta, además de ceñirse al estilo activo, también se pueden considerar como reflexivos, lo que demuestra que no se posee un estilo único al aprender. Es decir, puede existir la combinación de estilos de aprendizaje en una misma persona, y ser utilizados de acuerdo con la motivación de adquirir conocimiento.

En cuanto al estilo pragmático, se observa en individuos que: aplican las ideas, tienden a impacientarse cuando hay personas que teorizan, descubren el aspecto positivo de las ideas e intentan experimentarlas. Es práctico, directo, eficaz, realista, rápido, decidido y planificador (Cortés, 2017). La Tabla 3, evidencia los resultados sobre la aplicabilidad del conocimiento aprendido. Se observa que los valores más representativos, fueron siempre con un $69,5 \%$ en la UNAD, y casi siempre con un 49,5\% en Uniguajira. La opción siempre representa el $32,4 \%$ en Uniguajira, y la opción casi siempre representa el $28,6 \%$ en la UNAD. En menores valores, se presentan las opciones: a veces con 15,2\% en Uniguajira, y 1,9\% en la UNAD; casi nunca (con $1 \%$ en Uniguajira y $0 \%$ en la UNAD; y nunca con $1,9 \%$ en Uniguajira y $0 \%$ en la UNAD.

Tabla 3. Aprendizaje pragmático.

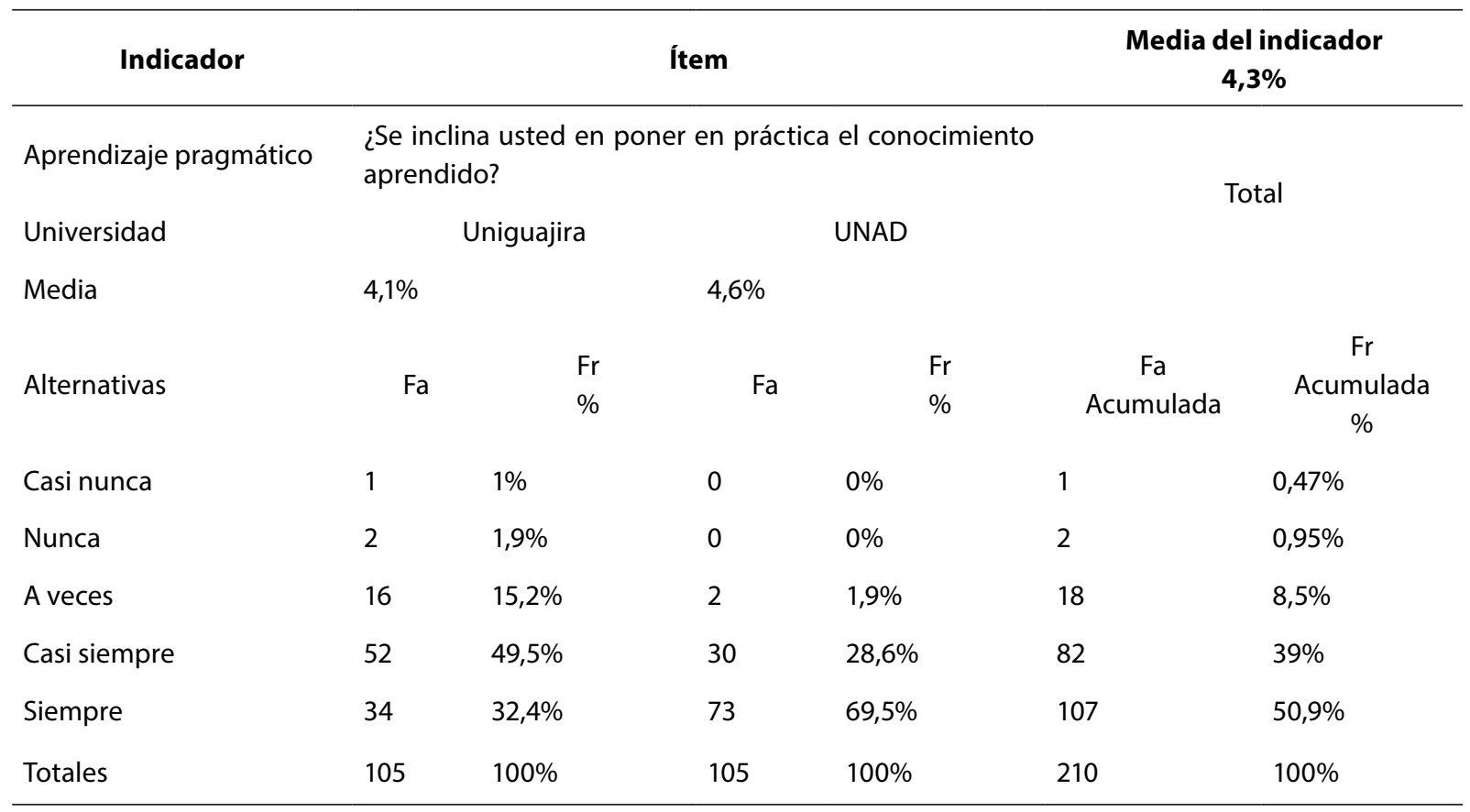


La tabla 3 indica que, de los 210 estudiantes indígenas encuestados, el 50,9\% siempre pone en práctica el conocimiento aprendido; el 39\% casi siempre practica el conocimiento aprendido. En menor proporción, el 8,5\% reconoce que a veces; el $0,95 \%$ nunca y $0,47 \%$, casi nunca aplica de manera práctica lo aprendido en teoría. La media de los datos arrojados en Uniguajira fue de $4,1 \%$ (porcentaje alto), y en la UNAD fue de $4,6 \%$ (porcentaje alto), esto en relación con el estilo de aprendizaje pragmático.

El valor de la media del indicador, arrojó una valoración del 4,3\%; de acuerdo con la significación en un rango del 1 al 5, se considera un porcentaje alto, en cuanto a poner en práctica el conocimiento aprendido (Tabla 3). Practicar el conocimiento aprendido, hace parte de los atributos del aprendizaje pragmático expuesto por Honey y Mumford (2000), quienes conside- ran que la principal competencia de las personas con predominancia en estilo pragmático, es la aplicación práctica de las ideas.

De acuerdo con Cortés (2017), el estilo teórico es el que adapta e integra las observaciones dentro de teorías lógicas y complejas, suele ser perfeccionista, analiza, sintetiza y busca la racionalidad y la objetividad. Es metódico, lógico, disciplinado y crítico. En la Tabla 4, se presentan los resultados del estilo de aprendizaje teórico, evidenciado en los estudiantes universitarios de la etnia wayuu. Al ser consultados sobre si adaptan e integran los hechos en teorías lógicas y complejas, el valor más representativo fue a veces con un $50,5 \%$ en Uniguajira, y casi siempre con un $58,1 \%$ en la UNAD. El valor de la opción siempre, representa el $13,3 \%$ en Uniguajira y $40 \%$ en la UNAD. La opción a veces, representa el 1,9\% en la UNAD; mientras que la opción casi nunca, representa el $4,7 \%$ en Uniguajira y $0 \%$ en la UNAD.

Tabla 4. Aprendizaje teórico.

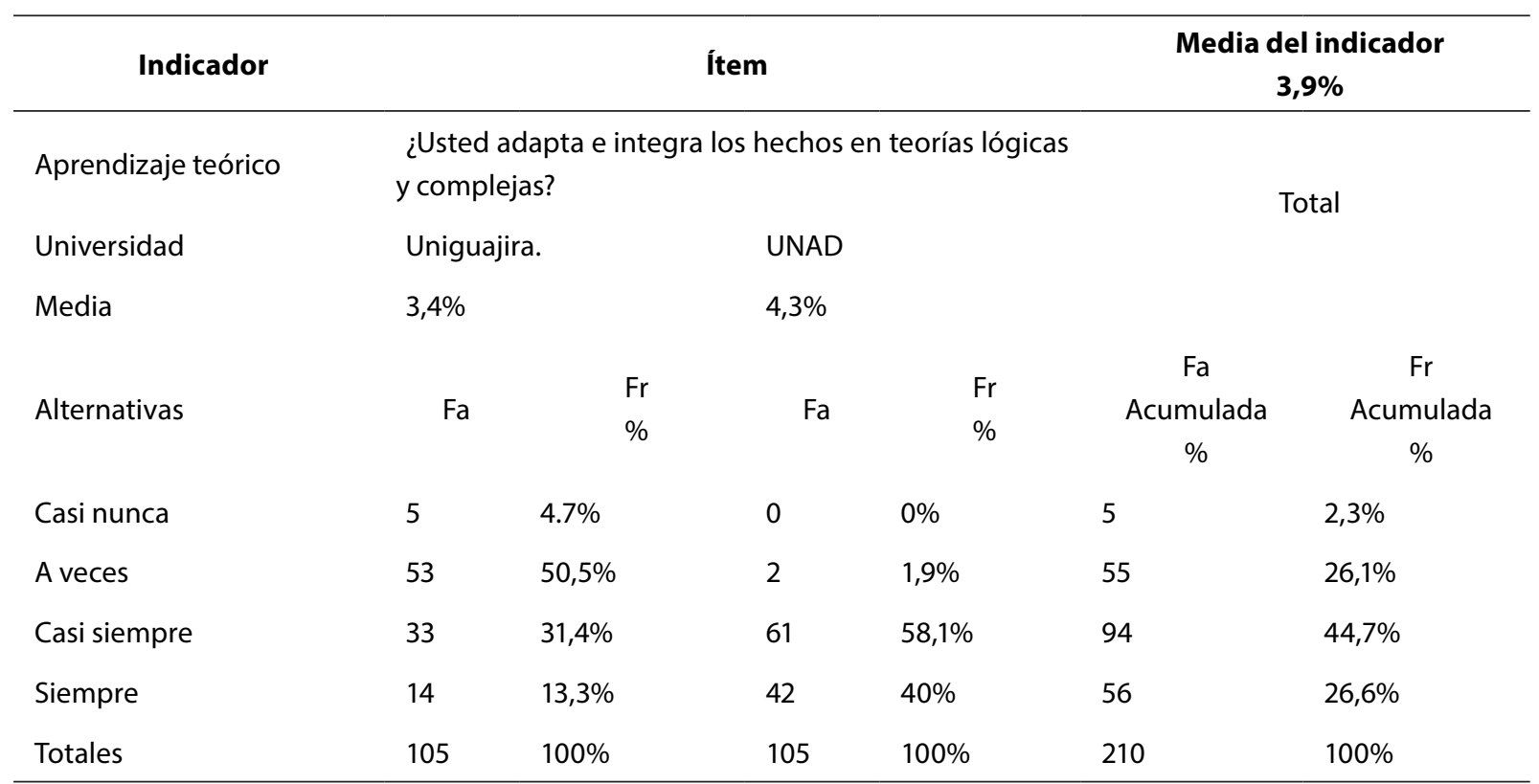

De los 210 estudiantes indígenas objeto de esta investigación: el 26,6\% siempre integra los hechos en teorías lógicas y complejas, el $44,7 \%$ casi siempre realiza la adaptación e integración de los hechos con las teorías. Igualmente, el 26,1\% reconoce que a veces lo hace y el 2,3\% casi nunca lo hace, y el 0,47\% nunca lo aplica (Tabla 4). La media de los datos en Uniguajira, representa un $3,4 \%$ (porcentaje moderado), mientras que en la UNAD es de $4,3 \%$ (porcentaje alto). El valor de la 
media del indicador arrojó un 3,9\%; de acuerdo con la significación en un rango del 1 al 5, se considera un porcentaje moderado, en cuanto a la integración que realizan los estudiantes de los hechos con las teorías coherentes, lógicas y complejas (Tabla 4).

Lo anterior permite dictaminar que los estudiantes wayuu de la UNAD, desarrollan en mayor proporción un estilo de aprendizaje teórico, en comparación con los estudiantes de Uniguajira. Sin embargo, un significativo porcentaje de la población encuestada hace menor uso del estilo de aprendizaje teórico, como medio principal para obtener el conocimiento. En otras palabras, este estilo de aprendizaje no es el más representativo o el de mayor uso entre la población encuestada, tal vez porque les resulta poco atractivo aprender en el contexto del estilo teórico, o quizás prefieran un lenguaje más práctico para interpretar los hechos que se vinculan con su área de formación.

\subsection{Discusión}

En el contexto de los resultados obtenidos, se puede afirmar que de alguna manera todos los estilos de aprendizaje se hacen evidentes en los estudiantes de la etnia Wayuu, destacándose: activo, reflexivo y pragmático, más que el teórico. No obstante, vale decir que los estilos no son interdependientes entre sí, ya que la relación entre ellos está en correspondencia con lo planteado por Bernabeu y Goldstein (2016), quienes definen los estilos, como: maneras preferidas, más no únicas ni excluyentes, para lograr el aprendizaje. Los resultados también son consistentes con lo esbozado por Lugo, Hernández y Montijo (2016), quienes manifiestan que todos los estilos están presentes en las personas en mayor o menor grado.

De acuerdo con Cardozo (2012), el estilo activo, uno de los más destacados en este trabajo, se encuentra relacionado con los demás, siendo positiva su relación con el estilo pragmático; lo que indica que ambos estilos podrían ser preferidos de manera moderada o alta por un mismo sujeto, con bastante frecuencia. A su vez, el estilo activo se relaciona en forma menos favorable con los estilos teórico y reflexivo, lo que pareciera indicar la poca compatibilidad entre ellos.

En el trabajo de Obando, Erazo y Carrillo (2019), quienes estudiaron los estilos de aprendizaje en escolares indígenas del Gran Mallama, se encontró una prevalencia del estilo de aprendizaje denominado auditivo. La diferencia de resultados con los de la presente investigación, radica más en el enfoque teórico abordado, que en los hallazgos establecidos durante el proceso. Esto ya que, en el análisis de Obando et al., se encuentran elementos en común, que también describen a los estilos de aprendizaje de los estudiantes de la Etnia Wayuu.

Otra investigación representativa, es la de Giordano, Prosek, Schmit y Wester (2020), quienes encontraron un importante nivel de abandono institucional por parte de las entidades educativas y gubernamentales, a los procesos de formación de los estudiantes nativo-americanos, quedando relegados a un grupo cuyo único nivel de formación es la brindada por la comunidad. En este sentido, es importante que, en Colombia y el resto de América, se fortalezca la participación institucional dentro del proceso formativo de los pueblos indígenas. Igualmente importante, es la unificación de los criterios para la determinación de los estilos de aprendizaje, lo cual facilitará la contrastación de los hallazgos, así como la posible realización de trabajos de intervención.

Es claro reconocer que la puesta en marcha de programas educativos permite una mejora significativa en la formación de los jóvenes, para que estos tengan la capacidad de desenvolverse en el mundo actual; el cual exige un alto nivel de competencias al insertarse dentro de la sociedad, como sujetos activos (Niebles, Martínez-Bustos \& Niebles-Núñez, 2020).

\section{Conclusiones}

En concordancia con los postulados de Honey y Mumford (2000), en este artículo se realizó una aproximación para identificar los estilos de 
aprendizaje que hacen parte del proceso educativo de los estudiantes indígenas wayuu, en las universidades públicas del departamento de La Guajira - Colombia. En tal sentido, los resultados permiten afirmar que las personas tienen estilos de aprendizaje diferentes; es decir, obtienen, procesan, almacenan, y recuperan la información y el conocimiento de manera distinta. Además, se puede señalar que las características culturales inciden en los estilos de aprendizaje que los individuos desarrollan. En este caso, los estudiantes de la etnia wayuu poseen una serie de rasgos particulares, que los hacen diferentes $y$, obviamente, esto influye y define la manera en que desarrollan sus procesos de aprendizaje.

En relación con los estilos de aprendizaje predominantes en esta investigación, se destacan con valores más altos, los estilos: activo, reflexivo y pragmático; y en menor proporción, se hace presente el estilo teórico, lo que puede ser consecuencia de las características de la población Wayuu. Sin embargo, se destaca el hecho de que cada individuo posee un modo preferente de abordar las tareas que le exige el entorno, estilo; lo cual no significa que no pueda hacer uso o desarrollar el aprendizaje mediante la aplicación de los otros estilos. Es decir, puede producirse o desarrollarse una combinación o alternancia de los diferentes estilos, para lograr un aprendizaje de alto valor en diversos momentos, contextos o circunstancias.

En el análisis de los elementos de la formación y aprendizaje de los estudiantes de pueblos nativos, existen grandes necesidades que pueden ser divididas en dos grupos: institucionales y conceptuales. Las primeras, se enfocan hacia la atención y la participación de los entes encargados de vigilar y fortalecer los procesos en las comunidades. Las conceptuales, se direccionan hacia las diferencias teóricas que existen al momento de estudiar los estilos de aprendizaje, lo cual implica la necesidad de unificar estos criterios para futuras investigaciones.

\section{Referencias}

Arias, F. (2012). El proyecto de investigación. Introducción a la metodología científica (5ta ed.). Caracas, Venezuela: Episteme.

Bartra, K. (2019). Estilos de aprendizaje de los estudiantes del ix ciclo de la escuela de lengua y literatura de la facultad de ciencias de la educación y humanidades-INICA, 2017. (Tesis de postgrado). Universidad Nacional de Huancavelica, Perú.

Bernabeu, N., \& Goldstein, A. (2016). Creatividad y aprendizaje: el juego como herramienta pedagógica. Madrid, España: Narcea Ediciones.

Bertrán, J. (2018). Cómo aprenden los seres humanos: Una aproximación psicopedagógica. Santiago de chile, Chile: Ediciones UC.

Camarero, F., Martín, F., \& Herrero, J. (2000). Estilos y estrategias de aprendizaje en estudiantes universitarios. Psicothema, 12 (4), 615-622. Recuperado de: http://www.psicothema.com/ pdf/380.pdf

Cardozo, A. (2012). Estilos y estrategias de aprendizaje ¿Constructos complementarios o diferentes? Estilos de aprendizaje: investigaciones y experiencias del V Congreso Mundial de Estilos de Aprendizaje. Santander, Colombia. Recuperado de: https://dialnet.unirioja.es/servlet/ articulo?codigo $=4636928$

Contreras, M., Salgado, F., Pina, F., \& Hernández, F. (2017). Enfoques de aprendizaje y enfoques de enseñanza: origen y evolución. Educación y Educadores, 20 (1), 5-15. http://dx.doi.org/10.5294/ edu.2017.20.1.4.

Cortés, M. (2017). Estilos de aprendizaje. Ciudad de México, México: Universidad Autónoma del Estado de Hidalgo.

DeBoer, G. (2019). A history of ideas in science education. New York, USA: Teachers College Press.

García, J., Santizo, J., \& Alonso, C. (2009). Uso de las TIC de acuerdo con los estilos de aprendizaje de docentes y discentes. Revista Iberoamericana de Educación, 48 (2), 1-14. Recuperado de: https:// rieoei.org/historico/deloslectores/2308Cue.pdf 
Giordano, A., Prosek, E., Schmit, M., \& Wester, K. (2020). "We Are Still Here": Learning from Native American Perspectives. Journal of Counseling \& Development, 98 (2), 159-171. https://doi. org/10.1002/jcad.12310

Gutiérrez, M., García, J., \& Melaré, D. (2012). Análisis Comparativo de los Estilos de Aprendizaje de diferentes grupos de alumnos de grado de la Escuela Universitaria de Magisterio. En Estilos de aprendizaje. Investigaciones y experiencias. Recuperado de: https://dialnet.unirioja.es/servlet/ articulo? codigo $=4679492$

Hernández, R., Fernández, C., \& Baptista, P. (2014). Metodología de la investigación (6ta ed.). México, D.F., Mexico: McGraw Hill.

Honey, P., \& Munford, A. (2000). Using your learning styles. Londres, Reino Unido: Maidenhead

Jonassen, D., \& Grabowski, B. (2012). Handbook of individual differences, learning, and instruction. Londres, Reino Unido: Routledge.

Lugo, C., Hernández, G., \& Montijo, E. (2016). Relación de los estilos y estrategias de aprendizaje con el rendimiento académico en estudiantes universitarios. Revista de estilos de aprendizaje, 9 (17), 268-288. Recuperado de: http://revistaestilosdeaprendizaje.com/article/view/1054

Niebles, W., Martínez-Bustos, P., \& Niebles-Núñez, L. (2020). Competencias matemáticas como factor de éxito en la prueba pro en universidades de Barranquilla, Colombia. Educación y Humanismo, 22 (38), 1-16. https://doi.org/10.17081/ eduhum.22.38.3590

Malacapay, M. (2019). Differentiated Instruction in Relation to Pupils' Learning Style. International Journal of Instruction, 12 (4), 625-638. https://doi. org/10.29333/iji.2019.12440a

Martin, A., Sperling, R., \& Newton, K. (2020). Handbook of Educational Psychology and Students with Special Needs. Londres, Reino Unido: Routledge.

Ministerio de Cultura de Colombia, Mincultura. (2019). Caracterizaciones de los Pueblos Indígenas de Colombia. Dirección de Poblaciones. Recu- perado de: http://www.mincultura.gov.co/ prensa/noticias/Documents/Poblaciones/ PUEBLO\%20WAY\%C3\%9AU.pdf

Montealegre, R. (2016). Controversias PiagetVygotski en psicología del desarrollo. Acta Colombiana de Psicología, 19 (1), 271-283. http:// www.dx.doi.org/10.14718/ACP.2016.19.1.12

Obando, A., Erazo, D., \& Carrillo, Á. (2019). Estilos de aprendizaje en escolares indígenas del Gran Mallama. Revista UNIMAR, 37 (2), 63-71. https:// doi.org/10.31948/Rev.unimar/37-2-art4

Organización Nacional Indígena de Colombia, ONIC. (2019). ONIC - Wayuu. Recuperado de: https://www.onic.org.co/debug/1156-wayuu

Pérez-Guardo, C., Tafur-Cabrera, J., MolinaPadilla, G., \& Hernández-Palma, H. (2019). Análisis del Papel del Educador en el Postconflicto. Un Acercamiento desde la Percepción de la Ciudad de Barranquilla, en Colombia. Información tecnológica, 30 (4), 199-208. http://dx.doi.org/10.4067/ S0718-07642019000400199

Pingo, G., Muñoz, C., Reyes, J., \& Polo, F. (2020). Teoría de la instrucción de Bruner y su incidencia en el aprendizaje significativo de los estudiantes del doctorado en educación. Diálogo, 43, 51-62. http://dx.doi.org/10.18316/dialogo.v0i43.6452

Riding, R., \& Rayner, S. (2013). Cognitive styles and learning strategies: Understanding style differences in learning and behavior. Londres, Reino Unido: Routledge.

Rodríguez, R. (2017). Los modelos de aprendizaje de Kolb, Honey y Mumford: implicaciones para la educación en ciencias. SOPHIA - Educación. 14 (1), 51-64. http://dx.doi.org/10.18634/ sophiaj.14v.1i.698.

Universidad de La Guajira, Uniguajira. (2019). Sitio oficial de Uniguajira. Recuperado de: http://www. uniguajira.edu.co/

Universidad Nacional Abierta y a Distancia, UNAD. (2019). Sitio oficial de la UNAD. Recuperado de: https://www.unad.edu.co/ 